Recepción: 07 / 11 / 2017

Aceptación: 23 / 01 / 2018

Publicación: 21 / 03 / 2018

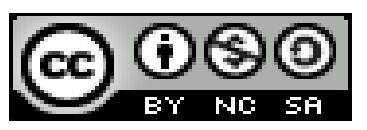

Ciencias de la computación

Artículo de Revisión

\title{
Las bitácoras virtuales en el aprendizaje de los contenidos de informática
}

\author{
The virtual logs in learning the contents of computer overview
}

\author{
Os logs virtuais no aprendizado do conteúdo do computador
}

\author{
David V. Romero-Jaén I \\ davidromero@hotmail.com \\ Jhonny M. Quiñonez-Quintero II \\ jhonnymquiñonez@hotmail.com \\ Carlos J. Reyes-Vera III \\ vera.carlos@hotmail.com
}

\section{Correspondencia: davidromero@hotmail.com}

\footnotetext{
I Ingeniero en Sistemas Informáticos, Tecnólogo en Computación, Docente de la Universidad Técnica Luís Vargas Torres de Esmeraldas, Esmeraldas, Ecuador.

II Magister en Ciencias de La Educación, Licenciado en Ciencias de la Educación Especialidad Informática Educativa, Ingeniero de Sistemas y Computación, Tecnólogo en Computación, Docente de la Universidad Técnica Luís Vargas Torres de Esmeraldas, Esmeraldas, Ecuador.

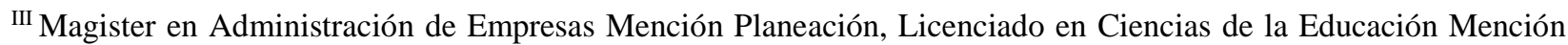
Informática Educativa, Tecnólogo en Informática, Docente de la Universidad Técnica Luís Vargas Torres de Esmeraldas, Esmeraldas, Ecuador.
} 


\section{Resumen}

Las bitácoras virtuales se constituyen en un formato que trabajan los alumnos de la modalidad presencial, en la que registran todos los materiales utilizados durante su proceso de aprendizaje. En atención a ello, se realizó un trabajo de investigación bajo un enfoque de corte descriptivo y de campo. El diseño de la investigación fue de carácter no experimental. La población de estudio la constituyeron 120 estudiantes de bachillerato del cantón Montufar en la provincia de Carchi del Ecuador. Para la recolección de la información se utilizó un instrumento tipo cuestionario con cinco (5) alternativas de repuestas. Los resultados indicaron que el 95\% de los encuestados consideran excelente la herramienta CmapTools, YouTube y el Mediamax, para la creación y publicación de mapas conceptuales con sus elementos enlazados a páginas web. Entre sus conclusiones se pudo constatar que las bitácoras virtuales como herramientas de aprendizaje de la informática por parte de los estudiantes de bachillerato fueron evaluadas como excelentes.

Palabras clave: bitácoras virtuales; aprendizaje, informática y estudiantes.

\section{Abstract}

The blogs virtual in the learning of the content of computer science overview virtual logs constitute a format serving the students of the modality, which recorded all materials used during the process of learning. In response to this, was a research paper under a descriptive Court and field approach. The research design was not experimental. Study population constituted the Montufar 120 students from canton high school in Ecuador Carchi province. For the collection of the information was used an instrument type questionnaire with five (5) choice of answers. The results indicated that $95 \%$ of respondents consider excellent tool CmapTools, YouTube and Mediamax, for the creation and publication of maps with its elements linked to web pages. Among its conclusions he could see that the virtual logs as learning tools of computer science by high school students were evaluated as excellent.

Keywords: virtual blogs; learning; computer science; and students.

\section{Resumo}

Os registros virtuais são constituídos em um formato em que os alunos da modalidade de sala de aula trabalham, no qual registram todos os materiais utilizados durante o processo de aprendizagem. Em resposta a isso, um trabalho de pesquisa foi realizado sob uma abordagem 
descritiva e de campo. O desenho da pesquisa foi de natureza não experimental. A população do estudo foi composta por 120 estudantes de bacharelado do cantão de Montufar, na província de Carchi, Equador. Um instrumento do tipo questionário com cinco (5) respostas alternativas foi utilizado para coletar as informações. Os resultados indicaram que 95\% dos respondentes consideram a ferramenta CmapTools, o YouTube e o Mediamax excelentes para a criação e publicação de mapas conceituais com seus elementos vinculados a páginas da web. Entre suas conclusões, constatou-se que os diários de bordo virtuais como ferramentas de aprendizado de computadores por estudantes do ensino médio foram avaliados como excelentes.

Palavras chave: logs virtuais; aprendizagem; computadores e estudantes.

\section{Introducción}

El siglo actual se ha visto marcado por una profunda revolución científico técnica que ha involucrado a las más diversas esferas de la actividad humana, y la educación no podía quedar al margen de este fenómeno. De esta manera los pedagogos se dedicaron a buscar aplicaciones didácticas a los diferentes recursos que la ciencia y la tecnología ponían en sus manos. Todo ello en consideración al uso de las Tecnologías de Información y Comunicación (TIC) en la vida cotidiana, pues la sociedad global demanda la conectividad, sistematización, almacenamiento y la digitalización de la información. Vivimos en la sociedad del conocimiento y la información, en donde aparece un nuevo concepto de alfabetización tecnológica asociada a las bitácoras virtuales como espacios ideales de aprendizajes para los estudiantes.

Dicha alfabetización tecnológica, viene sustituyendo la forma tradicional del proceso de enseñanza aprendizaje en el aula, en la que la incorporación de las tecnologías de la información a través del uso de las bitácoras virtuales como escenarios de aprendizaje, ha permitido la socialización entre estudiantes y docentes facilitando el intercambio de saberes a través de los espacios virtuales de enseñanza y de forma particular la enseñanza de los contenidos de informática. El estudio de la informática ha sido reconocido de gran utilidad a las exigencias actuales de comunicación de la sociedad, ante tal planteamiento en este artículo se identificaron los tipos de bitácoras virtuales de mayor uso entre los estudiantes de educación media. 


\section{Desarrollo}

La bitácora virtual, se refiere a sitios web actualizados periódicamente que recopilan cronológicamente textos o artículos de uno o varios autores donde lo más reciente aparece primero, con un uso o temática en particular, siempre conservando el autor la libertad de dejar publicado lo que crea pertinente. (Conejo 2002). Estos artículos por lo general incluyen la posibilidad de que los visitantes del blog añadan comentarios a los mismos, fomentando así la interacción entre el autor y el lector. El buscador de bitácoras Technorati.com es la mejor y más completa herramienta para encontrar blogs sobre cualquier tema.

Un blog es un sitio web en el que se va publicando contenido cada cierto tiempo en forma de artículos (también llamados posts) ordenados por fecha de publicación, así el artículo más reciente aparecerá primero. Uno de los principales motivos de la rápida expansión de este medio de comunicación ha tenido es la relativa facilidad que ofrecen para ser creados y manejados por cualquier usuario con conocimientos básicos de Internet aún sin tener conocimientos sobre el diseño de páginas web; esto ha hecho que todo aquel que quiera tener un espacio en la red para expresarse, haya encontrado en los blogs un medio ideal para hacerlo (Gallego Torres, 2006). La facilidad de su manejo, la posibilidad de compartir textos, imágenes y sonido, aunado a la interacción entre quien publica y los visitantes, convierte a los blogs en un poderoso recurso educativo al alcance de docentes y estudiantes.

Origen de Bitácora Virtual, weblog o Blog. El estadounidense Dave Winer escribió hace diez años el primer post de Scripting News, la primera weblog de la historia. El título de inventor del concepto blog se lo disputa Jorn Barger, un programador que asegura que creó los blogs cuando, en diciembre del año 1997, creó RobotWisdom.com, un batiburrillo de enlaces a artículos sobre cultura, libros y tecnología. Berger asegura que la web de Winer no es un blog, ya que tiene un orden cronológico confuso.

Elementos de las Bitácoras Virtuales, weblog o Blog. En un blog los artículos aparecen ordenados cronológicamente, pero en sentido inverso; lo más reciente es lo que se encuentra publicado en la parte superior de la página, inmediatamente después del título del blog. Todos los artículos están en una columna central y por lo general pueden leerse completos sin necesidad de acceder a otro enlace. 
También se puede observar una columna lateral con información extra; datos de identificación del autor, enlaces de interés a otras páginas y blogs, acceso al archivo de lo anteriormente publicado, buscador, Chat, álbum de fotos, libro de visitas, estadísticas; todo lo que el autor considere apropiado para la temática de la página o su gusto personal. La estructura básica de los artículos es similar a una noticia de prensa, con titular, fecha y cuerpo del artículo. En la parte inferior del mismo se puede encontrar el nombre del autor, la categoría, etiquetas (palabras claves) y un elemento que permite la interacción entre el autor y el lector: los comentarios.

Al igual que el resto de las utilidades del blog, la manera en que se van a manejar los comentarios puede ser definida por el autor. Por eso encontramos blogs que tienen acceso libre para comentar incluso de manera anónima y en otros hay que ser usuario registrado del servidor del servicio de blog. También el autor tiene la opción de moderar los comentarios que serán publicados, o solicitar una clave de confirmación para evitar comentarios automáticos o spam; lo que no puede el autor es limitar el libre acceso a su blog una vez creado; de ser esa su intención le estaría quitando la esencia al blog como medio de comunicación.

Elementos de las Bitácoras Virtuales, weblog o Blog. En un blog los artículos aparecen ordenados cronológicamente, pero en sentido inverso; lo más reciente es lo que se encuentra publicado en la parte superior de la página, inmediatamente después del título del blog. Todos los artículos están en una columna central y por lo general pueden leerse completos sin necesidad de acceder a otro enlace. También se puede observar una columna lateral con información extra; datos de identificación del autor, enlaces de interés a otras páginas y blogs, acceso al archivo de lo anteriormente publicado, buscador, Chat, álbum de fotos, libro de visitas, estadísticas; todo lo que el autor considere apropiado para la temática de la página o su gusto personal. Entre sus conclusiones se pudo constatar que las bitácoras virtuales como herramientas de aprendizaje de la informática por parte de los estudiantes de bachillerato fueron evaluadas como excelentes.

Al igual que el resto de las utilidades del blog, la manera en que se van a manejar los comentarios puede ser definida por el autor. Por eso encontramos blogs que tienen acceso libre para comentar incluso de manera anónima y en otros hay que ser usuario registrado del servidor del servicio de blog. También el autor tiene la opción de moderar los comentarios que serán publicados, o solicitar una clave de confirmación para evitar comentarios automáticos o spam; lo que no puede 
el autor es limitar el libre acceso a su blog una vez creado; de ser esa su intención le estaría quitando la esencia al blog como medio de comunicación.

Creación y publicación de una Bitácora Virtual, weblog o Blog. Para crear un blog se puede seleccionar entre los proveedores de servicio gratuito.

\section{Según Gallego Torres (2006) los más populares son:}

Blogger (www.blogger.com)

Bitácoras (www.bitacoras.com)

Blogalia (www.blogalia.com)

MyBlog (www.myblog.es)

Wordpress (www.wordpress.org)

Utilizando cualquier de estos proveedores se debe llenar un formato de registro, similar al que se utiliza para abrir una cuenta de correo electrónico; una vez registrado el usuario tiene la posibilidad de crear uno o más blogs, asignarle un título según la temática o el gusto del autor y acceder a las opciones del panel, desde el cual se administra el blog: creación de artículos, selección y edición de la plantilla, moderación de comentarios e incluso compartir el manejo del blog con otros autores. Además de esas opciones básicas, cada proveedor de blogs ofrece servicios particulares, una sección de ayuda y preguntas frecuentes, tutoriales e incluso un blog sobre el uso del servicio.

Complementos de uso para las Bitácoras Virtuales. Existen algunas herramientas que pueden servir de complemento para el diseño de estas herramientas tales como:

YouTube (www.youtube.com) Es un potente servidor que permite almacenar archivos audiovisuales. Genera un código que al ser incorporado a un artículo permite ver y escuchar videos desde el blog.

RadioBlogClub y GoEar (www.radioblogclub.com, www.goear.com)

Flickr (www.flickr.com) permite crear y publicar álbum de fotos. 
ImageShack (www.imagesahack.us) Servidor para almacenamiento de imágenes.

Cmap Tools (http://cmap.ihmc.us) Para crear y publicar mapas conceptuales de excelente presentación, permitiendo enlazar sus elementos con páginas web.

SlideShare (http://slideshare.net) Para publicar presentaciones en

Power Point y ser leídas dentro del cuerpo del artículo, totalmente en línea.

Mediamax (www.mediamax.streamload.com) Disco duro virtual de 25

GB en el cual se puede guardar cualquier formato de archivo, luego puede ser descargado desde el blog por los visitantes.

Meebome (www.meebome.com) Sitio web de mensajería instantánea, genera un portal de chat que puede ser incorporado al blog, permitiendo el intercambio de mensajes en tiempo real entre los visitantes y el autor cuando éste se encuentre conectado. Requiere registro solamente del autor.

Uso del Blog como recurso de enseñanza y aprendizaje. Conejo (2002) considera que el uso de los blogs en ambientes educativos solamente está limitado por la imaginación, mencionando las siguientes posibilidades:

\section{Opciones para educadores:}

Contenidos relacionados con la práctica profesional.

Compartir conocimiento personal y de la red.

Avisos, consejos educativos para estudiantes.

Anuncios de cursos, talleres, conferencias, eventos, etc.

Enlaces.

Administración de contenidos: textos, imágenes, audio, video. 


\section{Opciones para estudiantes:}

Reflexiones o diarios escritos. Registro.

Administración del conocimiento.

Presentación de tareas y asignaciones, revisión y evaluación de las mismas.

Diálogos con el grupo de trabajo.

Portafolios electrónicos.

Recursos compartidos relacionados con el curso.

Edublog es la palabra que ha surgido para distinguir a los blogs cuyo uso propuesto es la educación. Por lo tanto, se puede entender el término edublog como aquellos weblogs cuyo principal objetivo es apoyar un proceso de enseñanza - aprendizaje en un contexto educativo (Lara, 2005).

\section{Tanto la educación como los weblogs comparten una característica fundamental:}

Ambos conceptos pueden definirse como procesos de construcción del conocimiento los cuales se hacen posible gracias a la interacción del docente y estudiantes, grupos de docentes y grupos de estudiantes.

\section{En éste sentido, García Manzano (2006) hace mención a varias categorías o modalidades de uso de los blogs en diferentes situaciones de enseñanza y aprendizaje:}

Sistema de gestión de recursos didácticos: Es el tipo de edublogs más utilizados en tareas docentes. El profesor propone, como complemento a la clase presencial, una serie de actividades que el alumno debe desarrollar empleando los recursos disponibles en el blog. Sin embargo, el profesor debe ser consciente de que no se trata de hacer lo mismo de siempre sobre nuevos soportes, sino desarrollar estrategias didácticas novedosas aprovechando las características propias del blog como herramienta web.

Multiblogs de profesores: Un grupo de profesores puede crear una bitácora en la cual compartir experiencias educativas, estrategias y recursos. 
Multiblogs de alumnos: Experiencias colaborativas del alumnado centradas en temas o tareas que se desarrollan siguiendo varias líneas de trabajo; por ejemplo, elaboración de proyectos y blogs temáticos de una determinada materia.

Cuadernos de trabajo individual: Son blogs que maneja un único autor.

Viene a sustituir al cuaderno de clase, con la variante de estar disponible en Internet, poder ser visitadas y complementadas con aportes y comentarios de otros estudiantes y profesores. Al igual que un cuaderno de clase, el estudiante lleva un registro de notas, apuntes, comentarios a las clases y libros de texto, así como involucrarse determinadas tareas didácticas asesoradas por algún profesor.

Software de Programación: son aquellas herramientas que un programador utiliza para poder desarrollar programas informáticos. Para esto, el programador se vale de distintos lenguajes de programación.

\section{Metodología}

El estudio está concebido dentro del tipo de investigación descriptiva, según Hernández, Fernández y Baptista (2010), se considera descriptiva dado que solo se describieron los hechos tal cual como se presenten sin modificar ni alterar su naturaleza. Asimismo, la investigación fue de campo, puesto que los datos fueron tomados directamente de la realidad de donde ocurren los hechos, en concordancia con lo reseñado por Arias (2004), quien afirma que consiste en la recolección de datos directamente de los sujetos investigados, o de la realidad donde ocurren los hechos (datos primarios). El diseño de la investigación fue no experimental que, según Hernández, Fernández, y Baptista (2010) plantean que los estudios no experimentales, poseen como característica principal la no manipulación de las variables, tal es el caso de este artículo. La población de estudio la constituyeron 120 estudiantes de bachillerato del cantón Montufar en la provincia de Carchi del Ecuador. Para la recolección de la información se utilizó un instrumento tipo cuestionario con cinco (5) alternativas de repuestas: Excelente, bueno, regular, malo y muy malo, bajo una escala tipo Licker, que según Chávez (2007) se aplica para estudios sociales e instrumentos con varias alternativas de repuestas, el mismo fue sometido a la validez de expertos y el cálculo de la confiabilidad a través del estadístico de Alpha de Cronbach. 


\section{Resultados}

A continuación, se presentan los resultados del análisis de los datos que aportaron los estudiantes investigados, posterior a la aplicación del instrumento tipo cuestionario.

Tabla 1. Conocimiento acerca de las bitácoras virtuales

\begin{tabular}{|c|c|c|c|c|c|c|c|c|c|c|}
\hline & \multicolumn{2}{|c|}{ Excelente } & \multicolumn{2}{|c|}{ Bueno } & \multicolumn{2}{|c|}{ Regular } & \multicolumn{2}{|c|}{ Malo } & \multicolumn{2}{|c|}{ Muy malo } \\
\hline Bitácoras & Fr & $\%$ & $\mathbf{F r}$ & $\%$ & $\mathbf{F r}$ & $\%$ & Fr & $\%$ & Fr & $\%$ \\
\hline YouTube & 114 & 95 & 6 & 5 & 0 & 0 & 0 & 0 & 0 & 0 \\
\hline Radio Blog Club y Go Ear & 80 & 67 & 30 & 25 & 5 & 8 & 0 & 0 & 0 & 0 \\
\hline Flickr & 102 & 85 & 18 & 15 & 0 & 0 & 0 & 0 & 0 & 0 \\
\hline Image Shack & 110 & 92 & 10 & 8 & 0 & 0 & 0 & 0 & 0 & 0 \\
\hline Cmap Tools & 114 & 95 & 6 & 5 & 0 & 0 & 0 & 0 & 0 & 0 \\
\hline Slide Share & 112 & 93 & 8 & 7 & 0 & 0 & 0 & 0 & 0 & 0 \\
\hline Mediamax & 114 & 95 & 6 & 5 & 0 & 0 & 0 & 0 & 0 & 0 \\
\hline Meebome & 112 & 93 & 8 & 7 & 0 & 0 & 0 & 0 & 0 & 0 \\
\hline PROMEDIO & 108 & 89 & 12 & 10 & 0.6 & $\mathbf{0}$ & $\mathbf{0}$ & $\mathbf{0}$ & $\mathbf{0}$ & $\mathbf{0}$ \\
\hline MEDIA & \multicolumn{10}{|c|}{4,72} \\
\hline \begin{tabular}{|l} 
DESVIACIÓN \\
ESTÁNDAR
\end{tabular} & \multicolumn{10}{|c|}{0.08} \\
\hline MEDIANA & \multicolumn{10}{|c|}{4,68} \\
\hline
\end{tabular}

Fuente: Elaboración propia.

Se observa en la tabla 1, que el $95 \%$ de los encuestados consideran excelente la herramienta CmapTools, YouTube y el Mediamax, para la creación y publicación de mapas conceptuales con sus elementos enlazados a páginas web, para la asimilación del aprendizaje en clases y para el almacenamiento masivo de archivos para el uso de las bitácoras virtuales. Seguido del 5\% por igual para cada uno de ellos que señaló la alternativa bueno. La bitácora slideshare fue evaluada como excelente por el $93 \%$ de los investigados para la creación y publicación de presentaciones en meebome para el intercambio de mensajes en tiempo real y powerPoint en línea y dentro del cuerpo del blog y, mientras que el 7\% lo hizo para la alternativa buena. La herramienta imageshack registró que el $92 \%$ de los investigados consideró excelente el uso de imágenes a través de esta herramienta para el aprendizaje de la informática, mientras que el $8 \%$ restante la considera buena. Seguido por el indicador flick con el $85 \%$ indicó la alternativa excelente para la creación y publicación de álbumes, donde el restante $15 \%$ estimó como buena. Finalmente se midió la herramienta radioblog club y goear donde el $67 \%$ de los investigados considera 
excelente las herramientas para el almacenamiento de archivos de mp3, mientras que el $25 \%$ y $8 \%$ la valoran buena y regular respectivamente.

De igual modo, se observa que el valor de la media fue de 4,72 con una desviación estándar de 0,08 entre los investigados señalando que la misma puede variar entre las alternativas excelente y bueno. La mediana con un valor 4,68 se ubicó por encima de la media señalando que existe una tendencia de las opiniones hacia las alternativas altas de medición. Tales resultados reflejados conllevan a resaltar, la utilidad de los recursos y herramientas informáticos con base a las áreas de conocimiento necesarias de los estudiantes, permitiendo un aprendizaje constructivo, entendido y fluido entre sus necesidades de saber y la experimentación con sus propios medios, convirtiendo las bitácoras virtuales en potenciales escenarios para la investigación, socialización y compartir de experiencias significativas con fines pedagógicos.

\section{Conclusiones}

Fue posible evidenciar, en un porcentaje considerado que las bitácoras virtuales de YouTube, Radio Blog Club y GoEar, Flickr, Image Shack, Cmap Tools, Slide Share, Mediamax, Meebome, las evaluaciones como excelentes herramientas virtuales en la adquisición de sus conocimientos académicos para la informática.

Las bitácoras virtuales de RadioBlogClub y GoEar, Flickr e ImageShack fueron evaluadas como buenas indicándolas como una herramienta de menor uso durante el aprendizaje de la informática por parte de los investigados.

La evaluación de la media ponderada de las bitácoras virtuales como herramientas de aprendizaje de la informática por parte de los estudiantes de bachillerato investigados, permitió ubicarlas como excelentes, indicando que les permite el desarrollo de los weblogs o blogs, en la que recopilan información de todo tipo y pueden ser escritos por uno o más autores.

Se constató según la opinión de los investigados que las bitácoras virtuales, pueden ser actualizados periódicamente y permiten recopilar cronológicamente textos o artículos de uno o varios autores donde lo más reciente aparece primero, con un uso o temática en particular, siempre conservando el autor la libertad de dejar publicado lo que crea pertinente.

Se confirmó la facilidad de su manejo, la posibilidad de compartir textos, imágenes y sonido, aunado a la interacción entre quien publica y los visitantes, convirtiéndolas en un poderoso recurso de aprendizaje académico. 